\title{
Linear systems and Multiplicity of ideals
}

\section{Lê Dũng Tráng}

The "Abdus Salam" ICTP, Strada Costiera 11, 34014 Trieste, Italy

E-mail address: ledt@ictp.it

in memory of my friend Sevin Recillas

\section{Introduction}

A result of P. Samuel ([17] p. 186, Chap.II, Théorème 5) says that in a local noetherian ring $(\mathcal{O}, \mathfrak{M})$ of Krull dimension $d$ in which the residual field $k$ is infinite, the multiplicity of a $\mathfrak{M}$-primary ideal $I$ is equal to the multiplicity of an ideal $\left(x_{1}, \ldots, x_{d}\right)$ generated by some parameter sequence $x_{1}, \ldots, x_{d}$ contained in $I$. By a theorem of Rees ([16] p.142 Theorem 9.44), this implies that the ideals $I$ and $\left(x_{1}, \ldots, x_{d}\right)$ have the same integral closure in the ring $\mathcal{O}$.

In fact Samuel's proof shows that the elements of the parameter sequence can be chosen to be general elements of $I$, namely superficial elements of $I$.

An interesting consequence of Samuel's result is that, in the case the local ring $\mathcal{O}$ is a Cohen-Macaulay ring, e.g. a regular or a local complete intersection ring, the multiplicity of the ideal $I$ in $\mathcal{O}$ is the length of the $\mathcal{O}$-module

$$
\mathcal{O} /\left(x_{1}, \ldots, x_{d}\right)
$$

Using a geometric interpretation of the multiplicity by C. P. Ramanujam ([15]), we shall give a geometric way to calculate the multiplicity. We shall consider the particular case of a non-singular complex surface and give an example with a geometric proof of a result of Mumford, as it was suggested to the author by M.S. Narasimhan.

Most of this note is written in the language of complex analytic spaces (see [2] and [1]), but the results can be stated and proved in the case of schemes of finite type (see definition in [3] Chap. IV 1.6.1) over an infinite field with equicharacteristic local rings.

2000 Mathematical Subject Classification: 13H15, 14B05, 14H20, 14H50, 32S05, 32S10, 32S 15 


\section{Integral closures and blowing-ups.}

Let $(\mathcal{O}, \mathfrak{M})$ be a reduced complex analytic local ring and let $J$ be an ideal of $\mathcal{O}$. We say that an element $x$ of $\mathcal{O}$ is integral over the ideal $J$ if there is a relation

$$
x^{n}+\sum_{i=1}^{n} a_{i} x^{n-i}=0
$$

where $a_{i} \in J^{i}$.

Elements of $\mathcal{O}$ which are integral over $J$ form an ideal $\bar{J}$ in $\mathcal{O}$ which contains $J$. This ideal is called the integral closure of $J$ in $\mathcal{O}$.

We know that an ideal $J$ of $\mathcal{O}$ defines an order function $\nu_{J}$ defined by

$$
\nu_{J}(x):=\sup \left\{k, x \in J^{k}\right\} \in \mathbb{N} \cup+\infty
$$

for any $x \in \mathcal{O}$.

We can define

$$
\bar{\nu}_{J}(x):=\liminf \frac{\nu_{J}\left(x^{k}\right)}{k} \in \mathbb{N} \cup+\infty
$$

Notice that one can show that $\bar{\nu}_{J}(x)$ is in $\mathbb{Q}$ and not in $\mathbb{R}$, as its definition suggests.

Then, we have the important following theorem (see [11] Théorème 2.1 or $[7])$ :

1.1. Theorem. Let $(\mathcal{O}, \mathfrak{M})$ be a reduced analytic local ring, $J$ be an ideal of $\mathcal{O}$ and $x \in \mathcal{O}$. Denote by $(Z, z)$ a germ of complex analytic space such that $\mathcal{O}_{Z, z}=\mathcal{O}$. The following conditions are equivalent:

i) The element $x$ is integral over the ideal $J$;

ii) We have $\bar{\nu}_{J}(x) \geq 1$;

iii) There is a modification $\pi: \tilde{Z} \rightarrow(Z, z)$ such that the space $\tilde{Z}$ is normal and $J \mathcal{O}_{\tilde{Z}}$ is principal and $x \circ \pi$ is a section of $J \mathcal{O}_{\tilde{Z}}$.

iv) Let $\pi: \tilde{Z} \rightarrow(Z, z)$ be the normalized blowing-up of $J$, then $x \circ \pi$ is a section of $J \mathcal{O}_{\tilde{Z}}$.

On the other hand we have the following consequence of a theorem of D. Rees ([16] p.142 Theorem 9.44):

1.2. Theorem. Let $(\mathcal{O}, \mathfrak{M})$ be an analytic local ring which is an integral domain. Let $I \subset J$ be $\mathfrak{M}$-primary ideals of $\mathcal{O}$. Then, these ideals have the same multiplicity if and only if they have the same integral closure in $\mathcal{O}$.

The preceding theorems give us the important corollary: 
1.3. Corollary. Let I be a $\mathfrak{M}$-primary ideal of a reduced analytic local ring $(\mathcal{O}, \mathfrak{M})$ and let $x_{1}, \ldots, x_{d}$ a sequence of parameters in $I$ which generates an ideal $\left(x_{1}, \ldots, x_{d}\right)$ having the same multiplicity as the one of $I$. The normalized blowing-up of I equals the normalized blowing-up of $\left(x_{1}, \ldots, x_{d}\right)$.

Proof: Let $(Z, z)$ be a germ of reduced complex analytic space such that $\mathcal{O}_{Z, z}=\mathcal{O}$. From the theorem of Rees, it is enough to prove that the normalized blowing-up $\pi: \tilde{Z} \rightarrow(Z, z)$ of $I$ is also the normalized blowingup of the integral closure $\bar{I}$ of $I$ in $\mathcal{O}$. We have $\bar{I} \supset I$, so $\bar{I} \mathcal{O}_{\tilde{Z}} \supset I \mathcal{O}_{\tilde{Z}}$. Theorem 1.1 implies that $\bar{I} \mathcal{O}_{\tilde{Z}} \subset I \mathcal{O}_{\tilde{Z}}$, so

$$
\bar{I} \mathcal{O}_{\tilde{Z}}=I \mathcal{O}_{\tilde{Z}}
$$

Therefore $\bar{I} \mathcal{O}_{\tilde{Z}}$ is invertible and $\pi$ factorizes uniquely by $\sigma$ through the normalized blowing-up $\bar{\pi}: \bar{Z}^{\prime} \rightarrow(Z, z)$ of $\bar{I} \mathcal{O}$ :

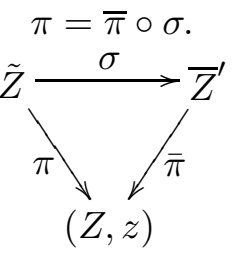

Now we show that $I \mathcal{O}_{\bar{Z}^{\prime}}=\bar{I} \mathcal{O}_{\bar{Z}^{\prime}}$. First, notice that $I \mathcal{O}_{\bar{Z}^{\prime}} \subset \bar{I} \mathcal{O}_{\bar{Z}^{\prime}}$, and, for $k \geq 0 I^{k} \mathcal{O}_{\bar{Z}^{\prime}} \subset \bar{I}^{k} \mathcal{O}_{\bar{Z}^{\prime}}$. By definition we have that $\bar{I} \mathcal{O}_{\bar{Z}^{\prime}}$ is locally principal. Since $\mathcal{O}_{Z, z}$ is noetherian, the ideal $\bar{I}$ is finitely generated. Let $f_{1}, \ldots, f_{k}$ be generators of $\bar{I}$. Let $y \in \bar{\pi}^{-1}(z)$. Since $\bar{I} \mathcal{O}_{\bar{Z}^{\prime}, y}$ is principal, one of the $f_{i} \circ \bar{\pi}$ 's, say $f_{1} \circ \bar{\pi}$, generates $\bar{I} \mathcal{O}_{\bar{Z}^{\prime}, y}$. On the other hand $f_{1}$ is integral over $I$, there is a relation:

$$
f_{1}^{N}+\sum_{1}^{N} a_{k} f_{1}^{N-k}=0
$$

where $a_{k} \in I^{k}$. Therefore in $\bar{I} \mathcal{O}_{\bar{Z}^{\prime}, y}$, we have:

$$
\left(f_{1} \circ \bar{\pi}\right)^{N}+\sum_{1}^{N}\left(a_{k} \circ \bar{\pi}\right) p\left(f_{1} \circ \bar{\pi}\right)^{N-k}=0
$$

and by dividing by $\left(f_{1} \circ \bar{\pi}\right)^{N}$ :

$$
1+\sum_{1}^{N} \frac{\left(a_{k} \circ \bar{\pi}\right)}{\left(f_{1} \circ \bar{\pi}\right)^{k}}=0
$$


which yields

$$
f_{1} \circ \bar{\pi}=-\sum_{1}^{N} \frac{\left(a_{k} \circ \bar{\pi}\right)}{\left(f_{1} \circ \bar{\pi}\right)^{k-1}} .
$$

Since $a_{k} \circ \bar{\pi}$ belongs to $I^{k}, a_{k} \circ \bar{\pi} \in \bar{I}^{k-1} I \mathcal{O}_{\bar{Z}^{\prime}}$ and we have, for $1 \leq k \leq N$,

$$
\frac{\left(a_{k} \circ \bar{\pi}\right)}{\left(f_{1} \circ \bar{\pi}\right)^{k-1}} \in I \mathcal{O}_{\bar{Z}^{\prime}, y}
$$

so $f_{1} \circ \bar{\pi} \in I \mathcal{O}_{\bar{Z}^{\prime}, y}$ and at $y$ :

$$
I \mathcal{O}_{\bar{Z}^{\prime}, y}=\bar{I} \mathcal{O}_{\bar{Z}^{\prime}, y}=\left(f_{1} \circ \pi\right) \mathcal{O}_{\bar{Z}^{\prime}, y} .
$$

Therefore the sheaf $I \mathcal{O}_{\bar{Z}}$ is invertible. It follows that $\bar{\pi}$ factorizes uniquely by $\tau: \bar{Z}^{\prime} \rightarrow \tilde{Z}$ through the morphism $\pi$ :

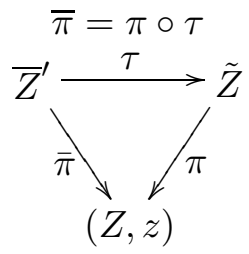

The uniqueness of the morphism implies that necessarily $\sigma$ is the inverse morphism of $\tau$, which shows that the normalized blowing-ups of $I$ and its integral closure $\bar{I}$ in $\mathcal{O}$ are the same.

\section{Geometry of Multiplicities}

In [15], C. P. Ramanujam gave an interesting geometrical interpretation of the multiplicity.

First recall that for an invertible sheaf $\mathcal{L}$ on a proper scheme $X$ (resp. on a compact analytic space), the Euler characteristic $\chi\left(\mathcal{L}^{n}\right)$ of the cohomology on $X$ of the $n$-th power $\mathcal{L}^{n}$ of $\mathcal{L}$ is a function of $n$ which coincides with a polynomial $P_{\mathcal{L}}(n)$ of degree $m \leq d:=\operatorname{dim} X$ in $n$. The coefficient of $n^{d}$ in this polynomial is

$$
\frac{1}{d !} d(\mathcal{L})
$$

and $d(\mathcal{L})$ is called the degree of $\mathcal{L}$.

In the case of local analytic rings the result of C.P. Ramanujam (see [15] Theorem p. 64 and Remark (1) p. 66) can be stated in the following way: 
2.1. Theorem. Let $(\mathcal{O}, \mathfrak{M})$ be a reduced local analytic local ring and $I$ a $\mathfrak{M}$-primary ideal of $\mathcal{O}$. Let $(Z, z)$ be a germ of analytic space such that $\mathcal{O}_{Z, z}=\mathcal{O}$. Let $\pi: Z^{\prime} \rightarrow(Z, z)$ be a bimeromorphic map such that $\pi^{*} I$ is an invertible sheaf on $Z^{\prime}$. The degree of the restriction of $\pi^{*} I$ to the space defined by $\pi^{*} I$ is equal to the multiplicity of the ideal $I$.

Considering the space defined by the coherent ideal sheaf $\pi^{*} I=I \mathcal{O}_{Z^{\prime}}$, we have the exact sequence

$$
0 \rightarrow I^{n+1} \mathcal{O}_{Z^{\prime}} \rightarrow I^{n} \mathcal{O}_{Z^{\prime}} \rightarrow I^{n} \mathcal{O}_{Z^{\prime}} \otimes_{\mathcal{O}_{Z^{\prime}}} \mathcal{O}_{Z^{\prime}} / I \mathcal{O}_{Z^{\prime}} \rightarrow 0
$$

which yields that the degree of the restriction of $\pi^{*} I$ to the space $\left\langle\pi^{*} I\right\rangle$ defined by $\pi^{*} I$ itself equals the degree of $I \mathcal{O}_{Z^{\prime}}$ because

$$
\chi\left(I^{n} \mathcal{O}_{Z^{\prime}}\right)-\chi\left(I^{n+1} \mathcal{O}_{Z^{\prime}}\right)=\chi\left(I^{n} \mathcal{O}_{Z^{\prime}} \otimes_{\mathcal{O}_{Z^{\prime}}} \mathcal{O}_{Z^{\prime}} / I \mathcal{O}_{Z^{\prime}}\right)
$$

and $\chi\left(I^{n} \mathcal{O}_{Z^{\prime}}\right)-\chi\left(I^{n+1} \mathcal{O}_{Z^{\prime}}\right)=P_{I \mathcal{O}_{Z^{\prime}}}(n)-P_{I \mathcal{O}_{Z^{\prime}}}(n+1)$ is a polynomial of degree $d-1$ with a term of degree $d-1$ equal to

$$
-\frac{1}{(d-1) !} d\left(I \mathcal{O}_{Z^{\prime}}\right) n^{d-1}
$$

Since $\chi\left(I^{n} \mathcal{O}_{Z^{\prime}} \otimes_{\mathcal{O}_{Z^{\prime}}} \mathcal{O}_{Z^{\prime}} / I \mathcal{O}_{Z^{\prime}}\right)$ has a term of degree $d-1$ equal to

$$
\frac{1}{(d-1) !} d\left(\left.I \mathcal{O}_{Z^{\prime}}\right|_{<\pi^{*} I>}\right) n^{d-1},
$$

Ramanujam's theorem implies

2.2. Corollary. The multiplicity of I equals:

$$
d\left(\left.I \mathcal{O}_{Z^{\prime}}\right|_{<\pi^{*} I>}\right)=-d\left(I \mathcal{O}_{Z^{\prime}}\right) \text {. }
$$

Ramanujam's theorem in particular applies to the cases when $\pi$ is the normalized blowing-up of $I$ or a resolution of $(Z, z)$ in which $\pi^{*} I$ is an invertible sheaf.

For instance, when the bimeromorphic map $\pi$ of the preceding theorem is a resolution of singularities $\pi$ of $(Z, z)$ for which $\pi^{*} I$ is invertible, we have:

2.3. Corollary. Assume that the map $\pi$ of the preceding theorem is a resolution of singularities for which $\pi^{*} I$ is invertible and $(\mathcal{O}, \mathfrak{M})$ is an integral domain, then the multiplicity of I equals $(-1)^{d-1}(D)^{d}$, where $d$ is the Krull dimension of $\mathcal{O}, D$ is the divisor defined by $\pi^{*} I$ on $Z^{\prime}$ and $(D)^{d}$ the d-th self-intersection of $D$.

Proof: According to Ramanujam's theorem the multiplicity of $I$ equals

$$
d\left(\left.I \mathcal{O}_{Z^{\prime}}\right|_{<\pi^{*} I>}\right) \text {. }
$$


The preceding corollary gives

$$
d\left(\left.I \mathcal{O}_{Z^{\prime}}\right|_{<\pi^{*} I>}\right)=-d\left(I \mathcal{O}_{Z^{\prime}}\right) .
$$

Let $D=<\pi^{*} I>$ be the divisor of $Z^{\prime}$ defined by the invertible sheaf $I \mathcal{O}_{Z^{\prime}}$. Hirzebruch-Riemann-Roch theorem (see $[\mathrm{H}]$ Theorem 4.1 Appen$\operatorname{dix} \mathrm{A})$ gives that the degree $d\left(I \mathcal{O}_{Z^{\prime}}\right)$ of $I \mathcal{O}_{Z^{\prime}}$ equals $(-1)^{d}(D)^{d}$. Precisely,

$$
\chi\left(I^{n} \mathcal{O}_{Z^{\prime}}\right)=\operatorname{ch}\left(I^{n} \mathcal{O}_{Z^{\prime}}\right) \operatorname{Todd}\left(\mathcal{T}_{Z^{\prime}}\right) \cap\left[Z^{\prime}\right]
$$

where $\left[Z^{\prime}\right]$ is the fundamental class of $Z^{\prime}$ and $\operatorname{ch}\left(I^{n} \mathcal{O}_{Z^{\prime}}\right)$ is the Chern character and $\operatorname{Todd}\left(\mathcal{T}_{Z^{\prime}}\right)$ is the Todd class of the tangent bundle of $Z^{\prime}$ :

$$
\operatorname{Todd}\left(\mathcal{T}_{Z^{\prime}}\right)=1+\frac{1}{2} c_{1}\left(\mathcal{T}_{Z^{\prime}}\right)+\ldots,
$$

and, since $I^{n} \mathcal{O}_{Z^{\prime}}$ is invertible, we have:

$$
\operatorname{ch}\left(I^{n} \mathcal{O}_{Z^{\prime}}\right)=1+n c_{1}\left(I \mathcal{O}_{Z^{\prime}}\right)+\ldots+\frac{1}{d !} n^{d} c_{1}^{d}\left(I \mathcal{O}_{Z^{\prime}}\right) .
$$

By comparing the terms of degree $d$ in $n$, for $n \gg 0$, on each side of the equality of Hirzebruch-Riemann-Roch theorem, we have:

$$
d\left(I \mathcal{O}_{Z^{\prime}}\right)=c_{1}^{d}\left(I \mathcal{O}_{Z^{\prime}}\right) \cap\left[Z^{\prime}\right] .
$$

Since $I \mathcal{O}_{Z^{\prime}}$ is $\mathcal{O}(-D)$ we have:

$$
c_{1}^{d}\left(I \mathcal{O}_{Z^{\prime}}\right) \cap\left[Z^{\prime}\right]=(-D)^{d}
$$

and the multipilicity of $I$ is $-d\left(I \mathcal{O}_{Z^{\prime}}\right)=-(-D)^{d}=(-1)^{d-1}(D)^{d}$.

\section{Linear Systems}

Let $\left(y_{1}, \ldots, y_{k}\right)$ be generators of an ideal $J$ of the reduced analytic local $\operatorname{ring} \mathcal{O}_{Z, z}$. We can construct the blowing-up of $J$ in the following way.

Let $Z$ be a representative of the germ $(Z, z)$ such that the germs $y_{i}$ $(1 \leq i \leq k)$ are defined by holomorphic functions defined on $Z$ also denoted by $y_{k}$ and let $Y$ be a representative of the support of $J$ in $Z$. Then on $Z \backslash Y$ we define the map $\lambda$ into the complex projective space $\mathbb{P}^{k-1}$ by:

$$
\lambda\left(z^{\prime}\right)=\left(y_{1}\left(z^{\prime}\right): \ldots: y_{k}\left(z^{\prime}\right)\right)
$$

for any $z^{\prime} \in Z \backslash Y$.

The graph $G$ of $\lambda$ is an analytic subspace of $Z \times \mathbb{P}^{k-1}$. The topological closure $\bar{G}$ of $G$ is naturally an analytic subspace of $Z \times \mathbb{P}^{k-1}$, because $G$ is the difference of the analytic set defined by

$$
\left(y_{1}: \ldots: y_{k}\right)=\left(u_{1}: \ldots: u_{k}\right)
$$

in $Z \times \mathbb{P}^{k-1}$, the $u_{i}$ 's are the homogeneous coordinates of $\mathbb{P}^{d-1}$, and the analytic set $Y \times \mathbb{P}^{k-1}$ (use e.g. Lemma 3.9 of [19]). One can show that 
the restriction to $\bar{G}$ of the first projection onto $Z$ is a representative of the blowing-up $p: Z_{J} \rightarrow(Z, z)$ of the ideal $J=\left(y_{1}, \ldots, y_{k}\right)$ in $(Z, z)$. Notice that $Z_{J}$ is reduced.

Let $n$ be the normalization of $\bar{G}$, then by corollary 1.3 the composition $p \circ n$ is also the normalized blowing-up $\pi: \tilde{Z} \rightarrow(Z, z)$ of $I$ in $(Z, z)$.

Consider the special case $J$ is generated by $d \geq 2$ generators where $d$ is the Krull dimension of $\mathcal{O}_{Z, z}$ and $Y=\{z\}$. The blowing-up $Z_{J}$ of $J$ is given in $Z \times \mathbb{P}^{d-1}$ by the equations

$$
u_{i+1} y_{i}-u_{i} y_{i+1}=0
$$

where $1 \leq i \leq d-1$. Therefore the second projection induces a map

$$
\lambda_{J}: Z_{J} \rightarrow \mathbb{P}^{d-1}
$$

which can be viewed as the family of curves defined by the linear system generated by $y_{1}, \ldots, y_{d}$. On the other hand the underlying set $\left|p^{-1}(z)\right|$ of the exceptional divisor of the blowing-up $p: Z_{J} \rightarrow(Z, z)$ is contained in $\{z\} \times \mathbb{P}^{d-1}$, so

$$
\left|p^{-1}(z)\right|=\{z\} \times \mathbb{P}^{d-1} .
$$

Let $\mathbf{a}=\left(a_{1}, \ldots, a_{d}\right)$ be a general point of $\mathbb{P}^{d-1}$. Since both $Z_{J}$ and $\mathbb{P}^{d-1}$ are reduced, the general fiber $\lambda_{J}^{-1}(\mathbf{a})$ is a general reduced curve in the linear system of curves generated by $y_{1}, \ldots, y_{d}$ (see [13]). Therefore, after normalization, the inverse image $n^{-1}\left(\lambda_{J}^{-1}(\mathbf{a})\right)$ is a non-singular (reduced) curve transverse to the exceptional divisor of the normalized blowing-up $p \circ n$. Since all the components of the exceptional divisor of $p \circ n$ project onto $\mathbb{P}^{d-1}$, the curve $\left.n^{-1}\left(\lambda_{J}^{-1} \mathbf{a}\right)\right)$ intersects all these components.

Apply these results to the case of a $\mathfrak{M}_{Z, z}$-primary ideal $I$ of the reduced analytic local ring $\mathcal{O}_{Z, z}$. The result of P. Samuel tells us that the ideal $I$ is integral over a ideal $J$ generated by $d$ general elements $x_{1}, \ldots, x_{d}$ of $I$, where $d$ is the Krull dimension of $\mathcal{O}_{Z, z}$. We have seen that the normalized blowing-up $\bar{\pi}: \tilde{Z} \rightarrow(Z, z)$ of $I$ coincides with the normalized blowingup of the ideal generated by $x_{1}, \ldots, x_{d}$. Let $\Gamma$ be a general curve in the linear system of curves generated by $x_{1}, \ldots, x_{d}$. From what precedes we observe that $\Gamma$ is reduced and the strict transform $\tilde{\Gamma}$ of $\Gamma$ by $\bar{\pi}$ is a nonsingular curve which intersects transversally all the components $D_{\alpha}, \alpha \in A$ of $\left|\bar{\pi}^{-1}(z)\right|$. This strict transform of a general curve in the linear system of curves generated by $x_{1}, \ldots, x_{d}$ can be obtained in the following way:

- Let $\pi_{J}: Z_{J} \rightarrow(Z, z)$ be the blowing-up of the ideal $J$. We have a map $\lambda_{J}: Z_{J} \rightarrow \mathbb{P}^{d-1}$ defined by the generators $x_{1}, \ldots, x_{d}$ of $J$. 
- Consider a general point $\mathbf{m}$ of $\mathbb{P}^{d-1}$, it is defined by $d-1$ linear equations $\sum_{i=1}^{d} \alpha_{i}^{j} \xi_{i}=0,1 \leq j \leq d-1$, where $\xi_{1}, \ldots, \xi_{d}$ are the homogeneous coordinates of $\mathbb{P}^{d-1}$.

- The fiber $\lambda_{J}^{-1}(\mathbf{m})$ of $\lambda_{J}$ over $\mathbf{m}$ is the strict transform by the blowing-up $\pi_{J}$ of the curve $\Gamma$ on $(Z, z)$ defined by $\sum_{i=1}^{d} \alpha_{i}^{j} x_{i}=0$, $1 \leq j \leq d-1$. Since $\mathbf{m}$ is a general point of $\mathbb{P}^{d-1}$, the germ of curve $(\Gamma, z)$ is a general curve in the linear system of curves generated by $x_{1}, \ldots, x_{d}$. The strict transform of $\Gamma$ by the normalized blowing-up $\bar{\pi}$ is $n^{-1}\left(\lambda_{J}^{-1}(\mathbf{m})\right)$.

Let $d_{\alpha}$ be the number of components of the strict transform $\tilde{\Gamma}$ which have a non-empty intersection with $D_{\alpha}$. Let $e_{\alpha}$ be the multiplicity of $D_{\alpha}$ in the divisor defined by $I \mathcal{O}_{\tilde{Z}}$. Then, we have:

3.1. Theorem. The multiplicity of the ideal I equals $\sum_{\alpha \in A} e_{\alpha} d_{\alpha}$.

Proof: Let $\varphi: \mathcal{Z} \rightarrow \tilde{Z}$ be a resolution of singularities of $\tilde{Z}$. The sheaf $(\bar{p} \circ \varphi)^{*} I \mathcal{O}_{\mathcal{Z}}=I \mathcal{O}_{\mathcal{Z}}$ generated by $I$ on $\mathcal{Z}$ is invertible. Let $D$ be the divisor of $\mathcal{Z}$ defined by $I \mathcal{O}_{\mathcal{Z}}$. According to corollary 2.3 , the multiplicity of $I$ equals $(-1)^{d-1}(D)^{d}$. We shall prove:

\subsection{Lemma.}

$$
(-1)^{d-1}(D)^{d}=\sum_{\alpha \in A} e_{\alpha} d_{\alpha}
$$

Using Ramanujam's result, this lemma obviously implies our theorem.

Proof of the lemma: First we observe that, since the image of $D$ by the map $\bar{p} \circ \varphi$ is a point $\{z\}$, we have

$$
D . d i v(f \circ \bar{p} \circ \varphi)=0,
$$

for any germ of functions $f \in \mathfrak{M}_{Z, z} \subset \mathcal{O}_{Z, z}$. In particular, if $f$ is a general element of the ideal $I$, we have:

$$
\operatorname{div}(f \circ \bar{p} \circ \varphi)=D+H(f)
$$

where $H(f)$ is the strict transform of $\{f=0\}$.

Now let us choose $\alpha_{i}^{j} \in \mathbb{C}$, such that the $d-1$ linear equations $\sum_{i=1}^{d} \alpha_{i}^{j} \xi_{i}=0,1 \leq j \leq d-1$, are general and define a general point of $\mathbb{P}^{d-1}$. Let $f_{j}:=\sum_{i=1}^{d} \alpha_{i}^{j} x_{i}=0,1 \leq j \leq d-1$. The functions $f_{j}$, $1 \leq j \leq d-1$, are general elements of the ideal $I$. The curve $\Gamma$ on $Z$ defined by $\left\{f_{1}=\ldots=f_{d-1}\right\}$ is a general curve in the linear system of curves generated by $x_{1}, \ldots, x_{d}$. The strict transform of $\Gamma$ by $\bar{p} \circ \varphi$ is the curve $H\left(f_{1}\right) \cap \ldots \cap H\left(f_{d-1}\right)$. 
The lemma will be consequence of the equality

$$
(-1)^{d-1}\left(D^{d}\right)=\left(D \cdot H\left(f_{1}\right) \ldots . H\left(f_{d-1}\right)\right) .
$$

In fact, since $\left(D \cdot D+H\left(f_{i}\right)\right)=0$, for $1 \leq i \leq d-1$, we have

$$
\left(D . H\left(f_{1}\right) \ldots . H\left(f_{d-1}\right)\right)=-\left(D \cdot H\left(f_{1}\right) \ldots . H\left(f_{d-2}\right) \cdot D\right)
$$

Therefore, by induction we can prove

$$
\left(D . H\left(f_{1}\right) \ldots . H\left(f_{d-1}\right)\right)=(-1)^{d-2}\left(D \cdot H\left(f_{1}\right) . D \ldots . D\right)=(-1)^{d-1}\left(D^{d}\right) .
$$

It remains to prove that $\left(D . H\left(f_{1}\right) \ldots . H\left(f_{d-1}\right)\right)=\sum_{\alpha \in A} e_{\alpha} d_{\alpha}$. The curve $\Gamma$ being a general curve in the linear system of curves generated by $x_{1}, \ldots, x_{d}$ the strict transform $\tilde{\Gamma}$ of $\Gamma$ by $\bar{\pi}$ is non-singular and transverse to the components of $\left|\bar{\pi}^{-1}(z)\right|$. Since $\varphi$ is a resolution of singularities $\tilde{Z}$ and

$$
\varphi^{-1}(\tilde{\Gamma})=H\left(f_{1}\right) \cap \ldots \cap H\left(f_{d-1}\right)
$$

the intersection points of $H\left(f_{1}\right) \cap \ldots \cap H\left(f_{d-1}\right)$ and $D$ are the inverse images by $\varphi$ of the intersection points of $\tilde{\Gamma}$ and $\left|\bar{\pi}^{-1}(z)\right|$ and the multiplicity $e_{\alpha}$ of $D_{\alpha}$ in $\tilde{Z}$ equals the multiplicity of the corresponding component in $\mathcal{Z}$. Since the intersection of $\tilde{\Gamma}$ with the divisor of $\tilde{Z}$ defined by $I \mathcal{O}_{\tilde{Z}}$ is $\sum_{\alpha \in A} e_{\alpha} d_{\alpha}$, we have

$$
\left(D . H\left(f_{1}\right) \ldots H\left(f_{d-1}\right)\right)=\sum_{\alpha \in A} e_{\alpha} d_{\alpha} .
$$

\section{An example}

Let us consider the simple case when $\mathcal{O}_{Z, z}$ is a regular local ring of Krull dimension 2. The multiplicity $e(I)$ of a $\mathfrak{M}_{Z, z}$-primary ideal $I$ is the multiplicity of an ideal generated $(f, g)$ by two general elements of $I$. Since $\mathcal{O}_{Z, z}$ is regular, it is Cohen-Macaulay, so:

$$
e(I)=\operatorname{dim}_{\mathbb{C}} \frac{\mathcal{O}_{Z, z}}{(f, g)} .
$$

Therefore, the multiplicity of $I$ is the intersection number of $f=0$ and $g=0$ at $z$.

The blowing-up $\pi_{J}$ of the ideal $J:=(f, g)$ gives the surface $Z_{J}$ defined by $\beta f-\alpha g=0$ in $Z \times \mathbb{P}^{1}$. The projection onto $Z$ restricted to $Z_{J}$ is the blowing-up $\pi_{J}$ and the projection onto $\mathbb{P}^{1}$ restricted to $Z_{J}$ extends to $Z_{J}$ the map $\lambda$ from $Z \backslash\{z\}$ into $\mathbb{P}^{1}$ defined by $\lambda\left(z^{\prime}\right)=\left(f\left(z^{\prime}\right): g\left(z^{\prime}\right)\right)$, for $z^{\prime} \in Z \backslash\{z\}$.

In [18] M. Spivakovsky shows that the singularities of the normalization $\tilde{Z}$ of $Z_{J}$ are rational. He calls these singularities Sandwich singularities (see also $[8])$. 
Let $\varphi: \mathcal{Z} \rightarrow \tilde{Z}$ be the minimal resolution of $\tilde{Z}$. The map $\pi_{J} \circ n \circ \varphi$, where $n$ is the normalization of $Z_{J}$, is a bimeromorphic map from a non-singular surface $\mathcal{Z}$ onto $Z$ :

$$
\mathcal{Z} \stackrel{\varphi}{\rightarrow} \tilde{Z} \stackrel{n}{\rightarrow} Z_{J} \stackrel{\pi_{J}}{\rightarrow}(Z, z) .
$$

Therefore, it is the composition of a sequence of point blowing-ups. In fact, since the strict transforms $H(f)$ and $H(g)$ of $f=0$ and $g=0$ by $\pi_{J} \circ n \circ \varphi$ are non-singular, distinct and transverse to $\left|\left(\pi_{J} \circ n\right)^{-1}(z)\right|$, the map $\pi_{J} \circ n \circ \varphi$ is an embedded resolution of the plane curve $f g=0$.

Conversally let $\sigma: \mathcal{Z}^{\prime} \rightarrow(Z, z)$ be the minimal embedded resolution of the germ of curve $\{f g=0\}$ in $(Z, z)$. Let $\mathcal{D}_{\alpha}, \alpha \in A$, be the components of the exceptional divisor $\mathcal{E}$ of $\sigma$ which intersect the strict transform of the curve $\{f g=0\}$. Consider the connected components of the closure of $\mathcal{E} \backslash \cup_{\alpha \in A} \mathcal{D}_{\alpha}$ and the singular surface $\tilde{Z}^{\prime}$ obtained from $\mathcal{Z}^{\prime}$ by contracting these components:

$$
\varphi^{\prime}: \mathcal{Z}^{\prime} \rightarrow \tilde{Z}^{\prime}
$$

Since $\sigma$ is the minimal embedded resolution of the germ of curve $\{f g=0\}$ in $(Z, z)$, the only components of $\mathcal{E}$ which might be of self-intersection -1 are among the components $\mathcal{D}_{\alpha}, \alpha \in A$. Therefore, the contraction $\varphi^{\prime}$ is the minimal resolution of $\tilde{Z}^{\prime}$.

The contraction of the components $\mathcal{D}_{\alpha}, \alpha \in A$, defines a holomorphic map:

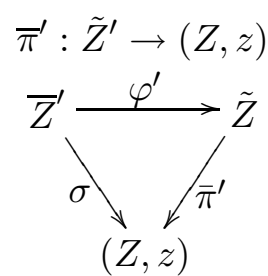

We have:

4.1. Lemma. The ideal sheaf $(f, g) \mathcal{O}_{\tilde{Z}^{\prime}}=\bar{\pi}^{\prime *}(f, g) \mathcal{O}_{Z}$ is invertible.

Proof: Let $\tilde{H}(f)$ and $\tilde{H}(g)$ be the strict transforms of $f=0$ and $g=0$ by $\bar{\pi}^{\prime}$, then the valuation along $\varphi^{\prime}\left(D_{\alpha}\right)$ of any function $h=u f+v g$ of $\mathcal{O}_{Z, z}$ being more that the one of $f$ or $g$, at any non-singular point $y$ of $\cup_{\alpha \in A} \varphi^{\prime}\left(D_{\alpha}\right)$ which is neither a singular point of $\tilde{Z}^{\prime}$ nor a point of $\tilde{H}(f)$ (resp. a point of $\tilde{H}(g)), f \circ \bar{\pi}^{\prime}$ (resp. $g \circ \bar{\pi}^{\prime}$ ) is a generator of $(f, g) \mathcal{O}_{\tilde{Z}^{\prime}, y}$.

On the other hand $f \circ \bar{\pi}^{\prime}$ (resp. $g \circ \bar{\pi}^{\prime}$ ) does not vanish on

$$
\tilde{Z}^{\prime} \backslash\left(\tilde{H}(f) \cup_{\alpha \in A} \varphi^{\prime}\left(D_{\alpha}\right)\right) \quad\left(\text { resp. } \tilde{Z}^{\prime} \backslash\left(\tilde{H}(g) \cup_{\alpha \in A} \varphi^{\prime}\left(D_{\alpha}\right)\right)\right) .
$$


Therefore, for any function $h$ in $(f, g)$, the meromorphic function $(h / f) \circ \bar{\pi}^{\prime}$ is bounded on

$$
\tilde{Z}^{\prime} \backslash\left(\tilde{H}(f) \cup \Sigma \cup \Sigma_{\tilde{Z}^{\prime}}\right)
$$

where $\Sigma$ is the finite set of singular points of $\cup_{\alpha \in A} \varphi^{\prime}\left(D_{\alpha}\right)$ and $\Sigma_{\tilde{Z}^{\prime}}$ is the finite set of singular points of $\tilde{Z}^{\prime}$. Since $\tilde{Z}^{\prime}$ is normal, this implies that $(h / f) \circ \bar{\pi}^{\prime}$ is holomorphic on $\tilde{Z}^{\prime} \backslash \tilde{H}(f)$. Similarly $(h / g) \circ \bar{\pi}^{\prime}$ is holomorphic on $\tilde{Z}^{\prime} \backslash \tilde{H}(g)$. It shows that the ideal sheaf $(f, g) \mathcal{O}_{\tilde{Z}^{\prime}}=\bar{\pi}^{\prime *}(f, g) \mathcal{O}_{Z}$ is invertible.

Thus, the contraction $\bar{\pi}^{\prime}$ factorizes uniquely through the normalized blowing-up

of the ideal $(f, g)$ :

$$
\bar{\pi}:=\pi_{J} \circ n: \tilde{Z} \rightarrow(Z, z)
$$

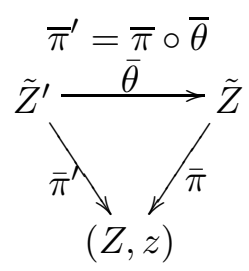

Since $\sigma: \mathcal{Z}^{\prime} \rightarrow(Z, z)$ is the minimal embedded resolution of $\{f g=0\}$ in $Z$, the map $\bar{\pi} \circ \varphi$ factorizes uniquely through $\sigma$ :

$$
\bar{\pi} \circ \varphi=\sigma \circ \eta \text {. }
$$

The map $\varphi^{\prime} \circ \eta$ is constant on the exceptional fibers of $\varphi$ and the space $\tilde{Z}$ is normal, so it gives a unique holomorphic map

$$
\bar{\eta}: \tilde{Z} \rightarrow \tilde{Z}^{\prime}
$$

such that $\bar{\eta} \circ \varphi=\varphi^{\prime} \circ \eta$.

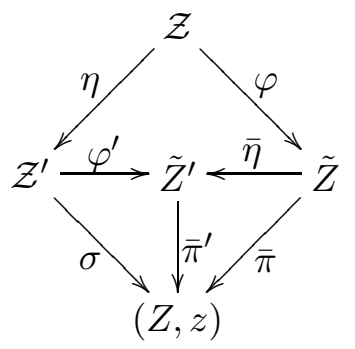

Necessarily, because of the uniqueness of the factorizations, $\bar{\eta}$ is the inverse of $\bar{\theta}$.

So, we have proved: 
4.2. Lemma. The map $\bar{\pi}^{\prime}: \tilde{Z}^{\prime} \rightarrow(Z, z)$ obtained by contraction is the normalized blowing-up of $I$.

The preceding results yields:

4.3. Theorem. Let $\left(\mathcal{O}_{Z, z}, \mathfrak{M}_{Z, z}\right)$ be the analytic local ring of the germ of a non-singular complex surface $(Z, z)$. Let $I$ be a $\mathfrak{M}_{Z, z}$-primary ideal of $\mathcal{O}_{Z, z}$. Consider $f$ and $g$, such that, the Milnor number of $f$ and $g$ at $z$ is minimum among the Milnor numbers at $z$ of the elements of $I$ and assume that the ideal $I$ is integral over the ideal $(f, g)$ generated by $f$ and $g$. The normalized blowing-up of $I$ in $(Z, z)$ is obtained from the minimal embedded resolution of the curve $f g=0$ by contracting the exceptional components which do not intersect the strict transform of $f g=0$.

Recall that the Milnor number of $f$ at $z$ (introduced in [12]) is a topological invariant of the germ of $f=0$ at $z$ (see [6] p. 261). It is the number of vanishing cycles of $f=0$ at $z$ and equals the first Betti number of $\{f=t\} \cap B_{\varepsilon}(z)$, where $B_{\varepsilon}(z)$ is a sufficiently small ball centered at $z$ and $\varepsilon \gg|t|>0$.

Elements $f$ with the minimum Milnor number in $I$ have the same topology by using the results of [9]. Moreover, since $f$ and $g$ belongs the linear system $\lambda f+\nu g$ and have the minimum Milnor number in this linear system, because $\lambda f+\nu g \in I$, one can show that they have the same embedded resolution (see e.g. [10] $\S 2$ ).

Theorem 4.3 indicates that one can choose superficial elements $(f, g)$ of $I$ to be elements of $I$ with minimum Milnor number at $z$ and such that $I$ and the ideal $(f, g)$ have the same multiplicity.

In this context Theorem 3.1 tells us that the multiplicity of $I$ equals the intersection number of the strict transform $\tilde{H}(f)$ of $f$ by the normalized blowing-up of $I$ and the exceptional divisor of this normalized blowing-up.

The preceding discussion also gives the following result:

Let $f$ be an element of $I$ having the smallest Milnor number at $z$. Let $\tau: \mathcal{Z} \rightarrow(Z, z)$ be the minimal embedded resolution of $f=0$.

4.4. Corollary. The ideal sheaf is $\tau^{*} I$ is invertible except possibly at the points where the strict transform of $f=0$ intersects the exceptional divisor of $\tau$. It becomes invertible after a sequence of blowing-ups which separates non-singular branches at these points.

Proof: As we indicate above, elements of $I$ with the minimum Milnor number have the same embedded resolution. So, the minimal embedded resolution of $f$ is also the minimal embedded resolution of $g$. However the minimal embedded resolution of $f$ might not be the minimal embedded resolution of $f g=0$, if the strict transforms of $f=0$ and $g=0$ in the 
minimal embedded resolution of $f=0$ have common points on the exceptional divisor, in which case one has to separate the strict transforms of $f=0$ and $g=0$, by a sequence of point blowing-ups to separate tangent non-singular branches.

We have already seen above that, in the embedded resolution of $f g=0$, the pull-back of $I$ is invertible. In fact, one can check that the points of the embedded minimal resolution of $f=0$, where the strict transforms of $f=0$ and $g=0$ have common points, are precisely the points where the pull-back of $I$ is not invertible.

In summary, the ideal $\tau^{*}(f, g)$ is invertible on the minimal embedded resolution of $f=0$ or on the modification of this minimal embedded resolution obtained by a sequence of point blowing-ups to separate the branches of $f=0$ and $g=0$ passing through common points on the exceptional divisor. Since this embedded resolution of $f g=0$ is non-singular, it is normal. So, it dominates the normalized blowing-up of $(f, g)$ which is also the normalized blowing-up of $I$ by corollary 1.3 .

M.S. Narasimhan showed me the following result of D. Mumford (see [14] Lemma p. 91-92) which can be obtained by using this viewpoint.

Consider $(Z, z)=\left(\mathbb{C}^{2}, O\right)$ and the ideal $I$ generated by the monomials $x^{r_{0}} y^{s_{0}}, \ldots, x^{r_{n}} y^{s_{n}}$. Let $\alpha=p / q$ ( $p$ and $q$ being relatively prime). Denote by $\nu_{\alpha}$ the discrete valuation of rank 1 on $\mathcal{O}_{\mathbb{C}^{2}, O}$ centered at $O$ such that

$$
\nu_{\alpha}\left(\sum_{i, j} a_{i, j} x^{i} y^{j}\right)=\min _{a_{i, j} \neq 0}(i p+j q) .
$$

4.5. Proposition. The exceptional divisors of the normalized blowing-up of $I$ are those prime divisors of the field of fractions of $\mathcal{O}_{\mathbb{C}^{2}, O}$ corresponding to valuations $\nu_{\alpha}$ with $\alpha=p / q$ where the least integer in the sequence of integers $r_{i} p+s_{i} q(0 \leq i \leq n)$ occurs at least twice.

Proof: First, notice that the ideal $I$ might not be primary for the maximal ideal $\mathfrak{M}_{\mathbb{C}^{2}, O}$ of $\mathcal{O}_{\mathbb{C}^{2}, O}$. However, there are unique integers $a$ and $b$ and a

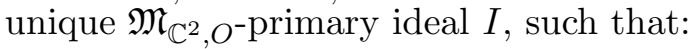

$$
I=\left(x^{a}\right)\left(y^{b}\right) I^{\prime} .
$$

Now, it is clear that, since the ideal $\left(x^{a}\right)$ and $\left(y^{b}\right)$ are invertible, the normalized blowing-up of $I^{\prime}$ is also the normalized blowing-up of $I$.

Let $x^{r_{0}^{\prime}} y^{s_{0}^{\prime}}, \ldots, x^{r_{n}^{\prime}} y^{s_{n}^{\prime}}$ be the generators of $I^{\prime}$, so, for $0 \leq i \leq n$ :

$$
r_{i}^{\prime}=r_{i}-a \text { and } s_{i}^{\prime}=s_{i}-b .
$$

As we have seen in our example above, the components of the normalized blowing-up of the $\mathfrak{M}_{\mathbb{C}^{2}, O}$-primary ideal $I^{\prime}$ come from components of the 
minimal embedded resolution:

$$
\pi: Z \rightarrow\left(\mathbb{C}^{2}, O\right)
$$

of $F G$, where $F$ and $G$ are two linear combinations:

$$
\sum_{i=o}^{i=n} \lambda_{i}^{j} x^{r_{i}^{\prime}} y^{s_{i}^{\prime}}, \text { with } j=0,1
$$

having the minimal Milnor number at $O$ and such that $I^{\prime}$ and $(F, G)$ have the same multiplicity. Precisely, consider the exceptional components $D_{\alpha}$, $\alpha \in A$, of this embedded resolution which intersect the strict transforms of $F G=0$. Now contract the exceptional components of $\pi$ which are not among the $D_{\alpha}$ 's. We obtain:

$$
\gamma: Z \rightarrow Z_{1}
$$

and $\pi$ defines a unique morphism $\pi_{1}: Z_{1} \rightarrow\left(\mathbb{C}^{2}, O\right)$, such that $\pi=\pi_{1} \circ \gamma$. We saw above that $\pi_{1}$ is the normalized blowing-up of $I$.

Corollary 4.4 suggests to consider first the minimal embedded resolution

$$
Z^{\prime} \rightarrow\left(\mathbb{C}^{2}, O\right)
$$

of $F=0$.

Consider the set $B$ of exponents $\left(r_{i}^{\prime}, s_{i}^{\prime}\right)$, for $0 \leq i \leq n$, in the real plane. The convex hull of $B$ is called the Newton Polyhedron of the set of exponents $B$. The Newton Polygon $\mathcal{N}(B)$ of $B$ is the set of faces viewed from the origin $(0,0)$. Call $A_{1}, \ldots, A_{\ell}$ the sides of the Newton Polygon $\mathcal{N}(B)$ with respective slopes

$$
-\frac{p_{1}}{q_{1}} \leq-\frac{p_{2}}{q_{2}} \leq \ldots \leq-\frac{p_{\ell}}{q_{\ell}}
$$

Notice that the slopes of the edges of $\mathcal{N}(B)$ are given by the linear forms $p \alpha+q \beta$ which attain their minimum at two exponents of $B$ at least.

Denote $F_{A_{j}}:=\sum_{\left(r_{i}^{\prime}, s_{i}^{\prime}\right) \in A_{j}} \lambda_{i} x^{r_{i}^{\prime}} y^{s_{i}^{\prime}}$ where the $\lambda_{i}$ are general complex numbers.

4.6. Lemma. In the linear family of polynomials

$$
F=\sum_{i=o}^{i=n} \lambda_{i} x^{r_{i}^{\prime}} y^{s_{i}^{\prime}}
$$

where $\lambda_{i} \neq 0$, for $0 \leq i \leq n$, are general complex numbers, the plane curve singularity $F=0$ at $O$ is isolated and has the topological type at $O$ of

$$
F_{1}=F_{A_{1}} \ldots F_{A_{\ell}} \text {. }
$$


Proof: Consider a polynomial $F_{0}(x, y)=\sum_{i=o}^{i=n} \beta_{i} x^{r_{i}^{\prime}} y^{s_{i}^{\prime}}=G_{A_{1}}+\ldots+G_{A_{\ell}}$ of this linear family, where:

$$
G_{A_{j}}=\sum_{\left(r_{i}^{\prime}, s_{i}^{\prime}\right) \in A_{j}} \beta_{i} x^{r_{i}^{\prime}} y^{s_{i}^{\prime}}
$$

The proof of Puiseux Theorem (see [19] Chap, IV §3) shows that the series:

$$
y-c x^{\gamma_{i}}-\ldots
$$

where $\gamma_{i}=q_{i} / p_{i}$ and $c$ is a root of $P_{i}(t):=\sum_{\left(r_{j}^{\prime}, s_{j}^{\prime}\right) \in A_{i}} \beta_{j} t^{s_{j}^{\prime}}$ divide $F_{0}$ in the ring $\cup_{n \geq 1} \mathbb{C}[y]\left[\left[x^{1 / n}\right]\right]$. For a general choice of the coefficients $\beta_{j}$, the polynomial $P_{i}$ has $b_{i}$ distinct solutions, where:

$$
b_{i}:=\sup _{\left(r_{j}^{\prime}, s_{j}^{\prime}\right) \in A_{i}} s_{j}^{\prime}-\inf _{\left(r_{j}^{\prime}, s_{j}^{\prime}\right) \in A_{i}} s_{j}^{\prime} .
$$

Therefore, for a general choice of the coefficients $\beta_{j}$, the Puiseux series are all distinct which implies that the product:

$$
\prod_{i=1}^{i=\ell} \prod_{P_{i}(c)=0}\left(y-c x^{\gamma_{i}}-\ldots\right)
$$

divides $F_{0}$ :

$$
F_{0}=u \prod_{i=1}^{i=\ell} \prod_{P_{i}(c)=0}\left(y-c x^{\gamma_{i}}-\ldots\right),
$$

where $u$ is a unit in $\mathbb{C}[[x, y]]$, because $\sum_{i=1}^{i=\ell} b_{i}=\sup _{\left(r_{i}^{\prime}, s_{i}^{\prime}\right) \in \mathcal{N}(B)} s_{i}^{\prime}$. This shows that $F_{0}=0$ has an isolated singularity at $O$ and:

$$
G_{A_{i}}=u_{i} \prod_{P_{i}(c)=0}\left(y-c x^{\gamma_{i}}-\ldots\right)
$$

where $u_{i}$ is a unit in $\mathbb{C}[[x, y]]$.

In particular, for a general choice of the $\beta_{i}$, each plane curve $G_{A_{i}}=$ 0 has an isolated singularity at $O$. Moreover, since $G_{A_{i}}$ is a weighted homogeneous polynomial, each branch of $G_{A_{i}}=0$ is also defined by a weighted homogeneous polynomial with the same weights. This implies that in each Puiseux series above has the simple form $y-c x^{\gamma_{i}}$. The Milnor number of these branches is $\left(p_{i}-1\right)\left(q_{i}-1\right)$ and their pairwise intersection numbers are $p_{i} q_{i}$.

One can also prove: 
4.7. Lemma. The minimum Milnor number of a linear combination of elements of $I^{\prime}$ equals the Kushnirenko number $2 S-a-b+1$, where $S$ is the area below the Newton polygon $\mathcal{N}(B)$, a is $\sup _{\alpha_{i} \neq 0} r_{i}^{\prime}$ and $b$ is $\sup _{\alpha_{i} \neq 0} s_{i}^{\prime}$.

Proof: The number of branches of $G_{A_{i}}=0$ at $O$ equals $b_{i} / q_{i}$ and the Milnor number each branch of $G_{A_{i}}=0$ at $O$ is $\left(p_{i}-1\right)\left(q_{i}-1\right)$. The pairwise intersection numbers of these branches are equal to $p_{i} q_{i}$.

Defining:

$$
a_{i}:=\sup _{\left(r_{j}^{\prime}, s_{j}^{\prime}\right) \in A_{i}} r_{j}^{\prime}-\inf _{\left(r_{j}^{\prime}, s_{j}^{\prime}\right) \in A_{i}} r_{j}^{\prime},
$$

the number of branches of $G_{A_{i}}=0$ at $O$ also equals $a_{i} / p_{i}$.

The pairwise intersection numbers of branches of $G_{A_{i}}=0$ and $G_{A_{j}}=0$, for $i<j$, are equal to $p_{i} q_{j}$.

So, the Milnor number $\mu\left(F_{0}, O\right)$ at $O$ of $F_{0}$ for a general choice of the coefficients $\beta_{i}$ equals (see [12] Theorem 10.5 and Remark 10.10):

$\sum_{i=1}^{i=\ell} \frac{b_{i}}{q_{i}}\left(p_{i}-1\right)\left(q_{i}-1\right)+\sum_{i=1}^{i=\ell} 2 p_{i} q_{i} \frac{b_{i}}{2 q_{i}}\left(\frac{b_{i}}{q_{i}}-1\right)+2 \sum_{i=1}^{i=\ell} \sum_{i<j} p_{i} q_{j} \frac{b_{i}}{q_{i}} \frac{b_{j}}{q_{j}}-\sum_{i=1}^{i=\ell} \frac{b_{i}}{q_{i}}+1$.

On the other hand:

$$
\begin{aligned}
2 S & =\sum_{i=1}^{i=\ell} \frac{b_{i}^{2}}{q_{i}^{2}} p_{i} q_{i}+2 \sum_{i=1}^{i=\ell-1} a_{i} b_{i+1} \\
a & =\sum_{i=1}^{i=\ell} a_{i} \quad \text { and } \quad b=\sum_{i=1}^{i=\ell} b_{i} .
\end{aligned}
$$

Using the equality:

we obtain:

$$
\frac{a_{i}}{p_{i}}=\frac{b_{i}}{q_{i}}
$$

$$
\mu\left(F_{0}, O\right)=2 S-a-b+1 .
$$

To finish the proof of Lemma 4.6, it is enough to notice that $F$ and $F_{1}$ belong to the same linear system and the minimum Milnor number is the minimum Milnor number among the analytic functions having the support of their Newton principal part on $\mathcal{N}(B)$, i.e. the Kushnirenko number (see [5] 1.10), as stated in the main theorem of Kushnirenko [5].

Now, the minimal embedded resolution of $F_{0}=0$ for a general choice of the coefficients $\beta_{i}$, is also an embedded resolution for $G_{A_{i}}=0$. We have noticed that the branches of $G_{A_{i}}=0$ are weighted homogeneous curves $\lambda x^{q_{i}}+\nu y^{p_{i}}=0$. This implies that the multiplicities of the coordinates 
$x$ and $y$ along the component intersected by the the strict transforms of the branches of $G_{A_{i}}=0$ are respectively equal to $p_{i}$ and $q_{i}$. Thus, this component defines a divisorial valuation of the field of fractions of $\mathcal{O}_{\mathbb{C}^{2}, O}$ given by $v_{i}(x)=p_{i}$ and $v_{i}(y)=q_{i}$. Therefore:

$$
v_{i}\left(\sum c_{\alpha, \beta} x^{\alpha} y^{\beta}\right)=\inf _{c_{\alpha \beta} \neq 0}\left(p_{i} \alpha, q_{i} \beta\right) .
$$

Each slope $-p_{i} / q_{i}$ of the Newton Polygon of $B$ defines such a valuation. By definition of the Newton Polygon, these valuations are defined by pairs of integers $(p, q)$, for which the minimum of the linear form $p \alpha+q \beta$ is obtained for at least two distinct pairs among $\left\{\left(r_{i}^{\prime}, s_{i}^{\prime}\right)\right\}$. These valuations correspond to the ones given by Proposition 4.5. To prove that these are the divisorial valuations of the exceptional components of the normalized blowing-up of $I^{\prime}$, it remains to prove that the minimal embedded resolution of $F_{0}=0$ already gives after contraction the normalized blowing-up of $I^{\prime}$.

As remarked before, we have to prove that the strict transform of a curve singularity $G=0$ defined by a general element $G$ of $I^{\prime}$, such that $I^{\prime}$ and the ideal $\left(F_{0}, G\right)$ have the same multiplicity, is disjoint from the strict transform of $F_{0}=0$ in the minimal embedded resolution of $F_{0}=0$.

To obtain this last assertion, notice that, in the minimal embedded resolution of $F_{0}=0$, the strict transforms of the branches $\lambda x^{q_{i}}+\nu y^{p_{i}}=0$ given by the edges of the Newton polygon for distinct $(\lambda: \nu)$ are disjoint. This implies that the strict transform of $G_{0}=0$, given by another general choice of the coefficients $\beta_{i}$, is disjoint from the strict transform of $F_{0}=0$.

As seen in $\S 3$ above, a general element $G$ of $I^{\prime}$ to be considered can be chosen as $G=G_{0}+H$, where $H$ is a general linear combination of monomial of $B$ which are not on $\mathcal{N}(B)$. The Puiseux series associated to $G$ are of the type $y-c x^{\gamma_{i}}+\ldots$. This shows that the strict transforms of the branches of $G=0$ intersect the strict transforms of the branches of $G_{0}=0$ in the minimal embedded resolution of $F_{0}=0$. This yields that the strict transforms of the branches of $G=0$ are disjoint from the strict transforms of the branches of $F_{0}=0$ in the minimal embedded resolution of $F_{0}=0$.

So, the normalized blowing-up of $I^{\prime}$ is already obtained from the minimal embedded resolution of $F_{0}=0$.

Therefore, the components of the minimal embedded resolution of $F_{0}=0$ intersected by the strict transforms of the branches of $F_{0}=0$ give the components of the normalized blowing-up of $I^{\prime}$. As we proved above, the divisorial valuations of the exceptional components of the normalized blowing-up of $I^{\prime}$ are effectively the valuations given in the Proposition 4.5.

This ends our proof. 


\section{References}

[1] W. Barth, K. Hulek, C. Peters, A. Van de Ven, Compact complex surfaces. Second edition. Ergebnisse der Mathematik und ihrer Grenzgebiete. 3. Folge. A (2004).

[2] Séminaire Cartan 1960/1961, Familles d'espaces complexes, Institut Henri Poincaré, Paris (1962).

[3] A. Grothendieck, Eléments de Géométrie Algébrique IV, Pub. IHES no 20 (1964).

[4] R. Hartshorne, Algebraic Geometry, Graduate Text in Maths 52, Springer-Verlag (1977).

[5] A. Kushnirenko, Polyèdres de Newton et nombres de Milnor, Inv. Math. 32 (1976) $1-31$.

[6] Lê Dũng Tráng, Calcul du nombre de cycles évanouissants d'une hypersurface complexe, Ann. Inst. Fourier 23 (1973), 261-270.

[7] Lê Dũng Tráng, Algebraic Methods in the Study of Singularities, Notes, Tokyo University, Komaba, 1977.

[8] Lê Dũng Tráng, Les singularités sandwich, in Resolution of singularities (Obergurgl, 1997), 457-483, Progr. Math., 181, Birkhäuser, Basel, 2000.

[9] Lê Dũng Tráng, C.P. Ramanujam, The invariance of Milnor's number implies the invariance of the topological type, Amer. J. Math. 98 (1976), 67-78.

[10] Lê Dũng Tráng, C. Weber, Equisingularité dans les pinceaux de Germes de Courbes Planes et $\mathcal{C}^{0}$-suffisance, Ens. Math. 43 (1997), 355-380.

[11] M. Lejeune-Jalabert, B. Teissier, Clôture intégrale des idéaux et équisingularité, Notes, Institut Fourier, Université de Grenoble. To appear in Ann. Univ. Toulouse. On web at: http://people.math.jussieu.fr/ teissier/old-papers.html

[12] J. Milnor, Singular points of complex hypersurfaces, Annals of Mathematics Studies, No. 61 Princeton University Press, Princeton, N.J. (1968)

[13] M. Morales, Polynôme de Hilbert-Samuel des clôtures intégrales des puissances d'un idéal $\mathfrak{M}$-primaire $\mathcal{P}$, Bull. Soc. Math. Fr. 112 (1984), 343-358.

[14] D. Mumford, Geometric Invariant Theory, Erg. der Math. und ihrer Grenzgebiete, Springer Verlag, Berlin-Heidelberg-New York, 1965.

[15] C.P. Ramanujam, On a Geometric Interpretation of Multiplicity, Inv. math. 22 (1973), 63-67.

[16] D. Rees, Lectures on the Asymptotic Theory of Ideals, LMS, LNS 113, 1988, Cambridge U.P.

[17] P. Samuel, La notion de multiplicité en Algèbre et en Géométrie algébrique, Journ. de Math. pures et appl. 30 (1951), 159-274.

[18] M. Spivakovsky, Sandwiched singularities and desingularization of surfaces by normalized Nash transformations, Ann. of Math. (2) 131 (1990), 411-491.

[19] H. Whitney, Tangents to an Analytic Variety, Ann. Math. 81 (1965), 496-549. 